\title{
ALADIN: AN INTERACTIVE DEEP SKY MAPPING FACILITY
}

\author{
Ph. PAILLOU, F. BONNAREL, F. OCHSENBEIN and M. CRÉZÉ \\ Centre de Données Astronomiques de Strasbourg - CDS \\ Observatoire Astronomique de Strasbourg \\ France
}

\begin{abstract}
Any astronomer, whether preparing observation runs or reducing data, requires access to information concerning the objects under investigation. Ideally the data should be from several wavelengths and should be as quantitative as possible. This leads to the concept of developing simultaneous interactive access to sky surveys, initially at optical wavelengths, and with state-of-the-art information systems for all field objects.

In order to satisfy this requirement, the CDS (Centre de Données astronomiques de Strasbourg) is developing a 'Deep Sky Mapping Facility' project, Aladin, which aims to connect the data stored at CDS (astronomical catalogues and Simbad), to pixel data from continuously updated deep optical sky surveys.
\end{abstract}

\section{Introduction}

Simply cross-correlating existing catalogues of astronomical data is not sufficient since CDS activities extend from radio wavelengths to gamma rays. Optical counterparts of these sources, if they exist, are not necessarily bright and referenced in a catalogue or database. From that consideration came the idea of a complete 'on-line' digitized sky atlas. This atlas must be available for all laboratories through networks and must be interactive. Such an atlas should allow any astronomer to point to a region of the sky for which he has some particular information, in order to receive the corresponding image (with accurate position and flux) and an overlay of all data archived at the CDS (Simbad content and catalogues archives) for that region.

For the CDS, this atlas is a crucial tool to continue the population of Simbad with faint objects and to perform a quality control of archived data (i.e. the contents of catalogues of Simbad). Using astrometrically calibrated images, it will be possible to project Simbad objects (or catalogued objects) in these fields to find quickly, for large numbers of objects, if there are any false positions. Likewise, using photometrically calibrated images, it will be possible to verify magnitudes, magnitude differences, or morphological types of objects contained in Simbad. Aladin can also be seen as an 'optical basis' allowing linking of various astronomical objects archived at the CDS and related at different wavelengths. This optical basis will be especially useful in verifying the correctness of new objects introduced in Simbad (cross-identification, coherency tests). Access to CDS catalogue archives and to pixel images will thus contribute to the automatic controlled integration of new data in Simbad (coordinates and flux comparison, use 
of images for difficult cases, etc.). One can also apply this method to verify the content of existing astronomical catalogues. Catalogues under construction could also be verified this way (error detection).

For the external user, Aladin offers the capability to use, in one's own laboratory, a service which is only partially available at one or two large institutes, for cross-identification purposes (detection of optical counterparts of X-ray, infrared or radio sources) or observations preparation (finding charts):

Optical counterparts of $X$-ray, infrared, gamma-ray, radio sources. We start with the image of a given sky region, and its astrometric calibration. It will then be possible to display, as overlay objects, positions coming from catalogues or from user's files. It will also be possible to represent error boxes associated with the positions of considered objects (a typical case for $\mathrm{X}$-ray sources). Furthermore, the user will see which objects are known in Simbad for the working region (special graphical symbols). Such objects can then be selected interactively in order to get related data (identifications, measurements, references). Aladin will then allow the detection of candidate optical counterparts with great efficiency and will also permit substantial savings in telescope time.

Observations preparation. The user will be able to select an observation field (centre and radius) in order to visualize it as it will appear at the telescope. In addition Aladin will use catalogue archives of the CDS and Simbad to provide information about which objects are known in this observation field, and through Simbad will give references about observations already done. Such a tool will especially be very useful for applications such as precise positioning for multi-objects spectroscopy (OPTOPUS, FUEGOS projects). In a more general way, this use of Aladin will constitute an essential tool for future observational astronomy; this is to be emphasized, as Simbad will contain the observations logs of some large instruments (ESO, CFHT, HST, IUE) and data from space missions (ROSAT, ISO) before the end of 1994.

For coming space experiments, Aladin removes the necessity of developing dedicated systems for each new mission. Likewise, 8-metre generation telescopes will benefit from more accurate and complete pointing data, in particular for multi-object spectroscopy.

\section{Aladin Project}

CDS began the Aladin project in 1992. The CDS council agreed with the general idea in September 1992. In December 1992, the Astronomy group of INSU/CNRS decided that a priority was the funding needed to start this CDS project. Funding for Aladin's construction was approved in June 1993, after a project review. Aladin will provide access, in addition to a complete sky atlas, to up-to-date data contained in Simbad and catalogue archives. Distribution of this tool to some selected sites is foreseen for the end of 1995.

For the interactive sky atlas, requirements in storage capacity are of the order of one Tbyte for complete sky coverage (1 TB for a complete sky in one colour with a resolution of 1 arcsec and coding on 16 bits; this can be reduced by using data compression). Hardware and software solutions for one Tbyte data archiving exist and are already in use. These solutions rely on 12" WORM optical disks systems and are now used in several places (ESA/ESRIN, STScI, NASA). The access speed to stored information is compatible with the proposed application (less than one minute to access a given sky region image). New technology to archive several tens of Tbytes 
has already been developed (optical tapes for instance). Software solutions foreseen for Aladin's achievement, as well from vendors point of view (DOROTECH, VRC Technologies) and that of CDS, allow a fast adaptation to these new technologies. The continuous development of information networks (Internet, Renater in France) and the use of adaptive image compression techniques will allow a fast remote access to archived data.

Considering surveys actually digitized now, several groups are likely to provide the CDS with sky images for the Aladin project, mainly the APS group from Minnesota University and the STScI. We decided to start with STScI digitized data, specifically including the integration of compressed SERC-J (factor 10) at the end of 1993, and that of compressed POSS-I E (factor 10) at the end of 1994, i.e. a complete digitized sky occupying about 100 Gbyte by the end of 1994 . From 1994, the CDS will also integrate some selected fields provided by the CAI (MAMA). In the future, it is thought to integrate the POSS-II survey digitized by STScI (from 1995) and CCD images generated by the LITE and DSS Sloan projects (from 1998).

Aladin will provide images of the complete sky. These images will allow one, when necessary, to perform processing or re-calibrations specifically adapted to the environment of a given object. This approach, ideally adapted for well-defined sky regions, is impossible when only using large catalogues. It will be facilitated by the modularity of Aladin, both with regard to new data integration possibilities (Hipparcos, Tycho, CCD images) and to reduction techniques. Moreover, Aladin will bring significant contributions to large catalogues now being produced, as follows:

1) Catalogue cross-identification: Aladin is perfectly adapted to compare catalogue archives of the CDS with the content of large catalogues. It will also be possible to use image pixels to verify (for difficult cases) the presence or the nature of an object mentioned in a catalogue.

2) Access to catalogue data: the CDS, with its experience in database development, will start a study concerning the management of large catalogues in database mode. Management of such a huge number of objects (up to one billion) needs the development of new methods.

\section{Project Achievement}

First studies have already been conducted for the Aladin project. These studies concern all aspects of the project: scientific requirements, technical choices for the archiving system, organization of the information stored, software developments (graphical user interface, data servers), and contacts with other institutes involved in image production and processing which could collaborate on this project. In parallel with these preliminary efforts, a prototype of Aladin has been constructed.

Figure 1 shows a simplified scheme of the functional software architecture of Aladin. This architecture is based on client-server philosophy which, on one hand, allows one to fully exploit advantages of a computer network (access to distributed computing resources) and, on the other hand, offers a modularity and a flexibility which facilitate the hardware and software evolutions of the system. Each CDS data source (images, catalogues, Simbad) is accessed through its dedicated server. The access to all these data from outside, through the international network, is managed by the 'CDS multimedia server', which is connected to each dedicated server through the CDS local network. The communication protocol and the functionalities associated to each dedicated server will be standardized. Each user of Aladin will then be connected to the CDS multimedia server through the international network and will be able to access all available data 


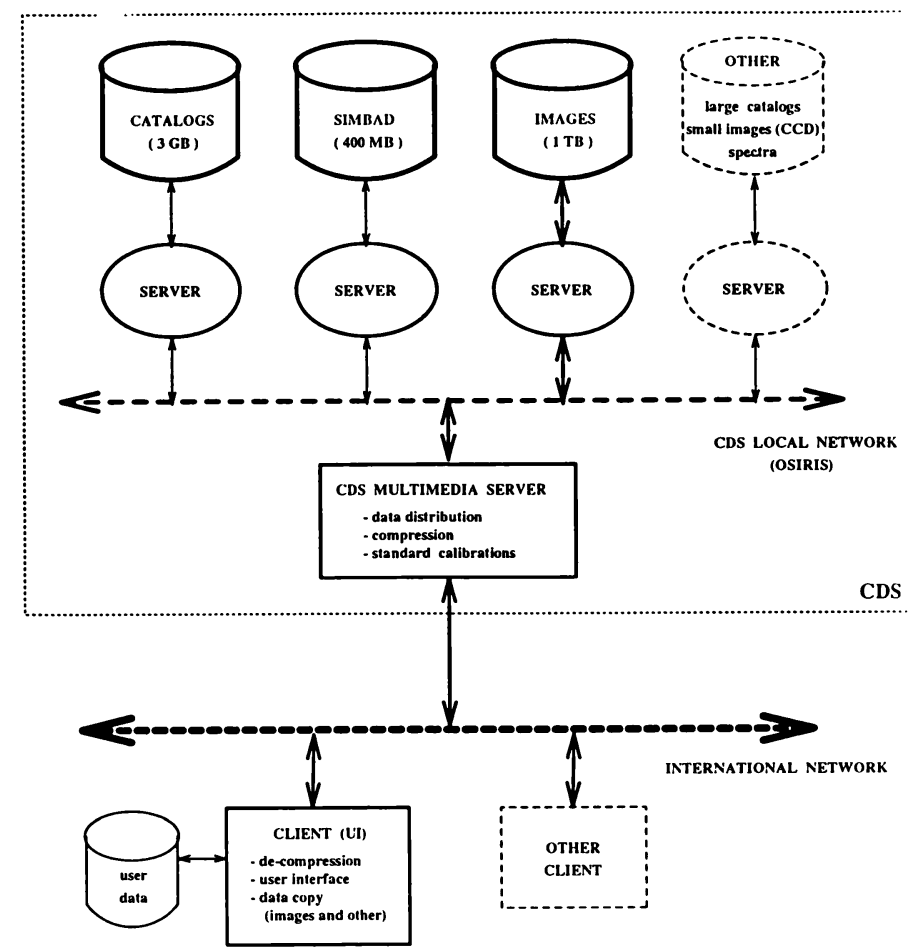

Figure 1. Functional software architecture of Aladin (client-server model).

stored (images and others).

We stress here development points which are essential for the project. These different points were gathered into two categories:

1) the CDS own developments. This work constitutes the 'kernel' of Aladin: data (images) integration, implementation of an image archiving system, multimedia server development for CDS data access (images, catalogues, Simbad and others), image server development, and connection to Simbad and catalogues archives;

2) the necessary tools. The following essential tools are required for the achievement of a functional product: image compression, astrometric and photometric calibrations of images, object detection and classification in images, interactive graphical user interface. The related developments can be done in collaboration with other groups.

We plan to provide Aladin's users with some elements for astrometric and photometric data calibration. These calibrations are divided into three levels:

- Level 0: use of results of calibration procedures (position and/or object magnitude) associated with archived pixel data, e.g. the STScI and the CAI (MAMA) procedures to perform a transformation from pixel coordinates to astronomical coordinates. 
- Level 1: data from astronomical catalogues or from Simbad is used to perform a photometric and/or an astrometric calibration of a well defined region of the sky (local calibration). At this level, the evolution of data archived at the CDS allows us to use standards of increasing quality to get better and better results (Guide Star Photometric Catalog II, Hipparcos data, Tycho data, etc.).

- Level 2: the user copies data of interest on his local computer (pixels, catalogues data, Simbad data) and uses his specific calibration procedures.

Distribution of a functional version of Aladin is foreseen for the end of 1995 . Several milestones were defined: southern hemisphere integrated in Aladin for the end of 1993, northern hemisphere integrated in Aladin for the end of 1994, distribution of version 1.0 of the user interface for the end of 1995.

\section{The Prototype}

A prototype of Aladin is being developed at the CDS, using some well defined test regions of the sky: the Large Magellanic Cloud and the Galactic anti-centre. The corresponding images are stored on the ATG optical disk system ( 9 Giga bytes) of the CDS.

For the LMC ( $\left.\alpha=5^{h} 24^{m n}, \delta=-69^{\circ} 48^{\prime}\right)$, J. Guibert (CAI-MAMA) digitized ESO plates in two colours, B and R (ESO 8968a, ESO 8967a). Each image is made up of 29,000 x 29,000 pixels coded on 16 bits, that is $1.5 \mathrm{~GB}$.

It is already possible to visualize the images of these two ESO plates of the LMC, and to display overlay GSC stars and Simbad objects using an interactive graphical user interface, GIFAD. The astrometric calibration was performed by the CAI using PPM data and allows one to interactively measure angular distances between objects with an accuracy better than one arcsec. The user can also display as overlay objects positions coming from its own data files (visualisation of infrared and $X$ sources for example).

The use of Aladin's prototype has already started at the Strasbourg Observatory for crossidentification of ROSAT X-ray sources in the Large Magellanic Cloud (M. Pakull). 